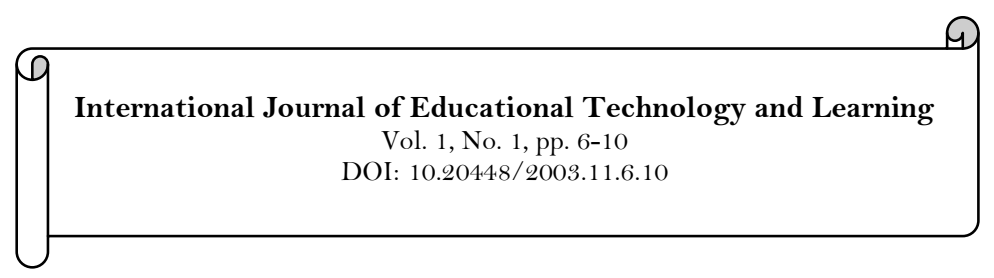

\title{
How to Teach the Sequential Part of Digital Electronics Basis with Project Pedagogy? Thanks to a Self-Working Card Trick Named “Cyclic Number”!
}

\author{
Pierre SCHOTT \\ ${ }^{1}$ Art et Recherche NUMérique (ARNUM), Ecole Supérieure d'Informatique, d'Electronique et d'Automatisme (ESIEA), Paris, \\ France.
}

\begin{tabular}{|c|c|}
\hline Abstract & \\
\hline $\begin{array}{l}\text { Why use Magic for teaching digital electronics theory and software? } \\
\text { Magicians know that, once the surprise has worn off, the audience } \\
\text { will seek to understand how the trick works. The aim of every } \\
\text { teacher is to interest their students, and a magic trick will lead them } \\
\text { to ask how? And why? And how can I create one myself? Whatever } \\
\text { the student's professional ambitions, they will be able to see the } \\
\text { impact that originality and creativity have when combined with an } \\
\text { interest in one's work. The students know how to "perform" a magic } \\
\text { trick for their family and friends, a trick that they will be able to } \\
\text { explain and so enjoy a certain amount of success. Sharing a } \\
\text { mathematical / informatics demonstration not easy and that they do } \\
\text { so means that they will have worked on understood and are capable } \\
\text { of explaining this knowledge. Isn't this the aim of all teaching? In } \\
\text { this article I present a self-working magic card trick, its global study } \\
\text { and how, with a project pedagogy, digital electronics can be teached. } \\
\text { I have presented, in a previous article in the same journal, all the } \\
\text { notions of combinatory digital electronics. In this article I presented } \\
\text { how to introduce the flip-flop gates, synchronous and asynchronous } \\
\text { counters designed thanks to transition tables without using } \\
\text { clear/ reset entries of the gates. In order to visualize the results, } \\
\text { seven segment displays with the associated encoders and decoders } \\
\text { could be introduce and simulated by an open source software named } \\
\text { "Logisim". The truth table of each command circuits of the complete } \\
\text { cycle can be calculated by Open Office. }\end{array}$ & $\begin{array}{l}\text { Keywords: } \\
\text { Higher education } \\
\text { Engineer } \\
\text { Educational method } \\
\text { Digital electronics software } \\
\text { Combinatory circuits } \\
\text { Sequential circuits } \\
\text { Project pedagogy } \\
\text { Magic } \\
\text { Logisim } \\
\text { Excel. }\end{array}$ \\
\hline
\end{tabular}

\section{Introduction}

I am fascinated by Magic (or rather conjuring!) and for many years I have used this way of teaching, both in my physics classes and as higher education teacher trainer.

I present in this paper firstly all the sequential digital electronics notions I can teach with the cyclic number, secondly the project I took and I have exposed and finally its answer and the sequential notions I have teached with it.

The aim of the magician is to hide the principles he uses (using Maths, Physics, Psychology, sleight of hand, etc...) by disguising the trick so that the audience has no way of discovering how it is done thus allowing the Magic to remain.

\section{The Teacher can do Exactly the Opposite: Unraveling a Magic Trick to Highlight the Principles Used! \\ 2.1. Summary}

In a previous paper (Schott, 2016) I presented the way to introduce with this magic trick the following combinational theory and electronics devices:

- Binary number system and weighted codes;

- Transcoders : binary <-> weighted code;

- Logic minimization using Karnaugh map (for example to synthesize these transcoders using the gates AND, OR, NOT); 
- Synthesize comparators thanks to other gates (NOT, NOR) and/or by arithmetic circuits;

- Seven segment displays with the associated encoders and decoders;

- Multiplexers and demultiplexers;

- An open source software named "Logisim";

In this paper I presented the way to introduce, with this magic trick, the following sequential theory an electronics devices. We will make students gather information about all these following notions at the time they need to, in order to keep going forward in their project:

- D-Latch, Flip-Flop Gate;

- Their truth table and transition table;

- Synchronous counters and command circuits.

\subsection{My Motivations to Propose Such Kind of Course}

I teached since many years with a descending method the introduction of digital electronics lecture. But the subject of the final work, I gave to the students, was always a peculiar subject such as a music partition, fitness program or the cyclic number card trick.

I thought I can build my lecture on this subject with an ascending method like the pedagogy project!

I chose the topic of magic, a subject which fascinates me, to be virtually certain that the topic would be completely new for the students. So they are in an unfamiliar situation. They will thus have to implement all the necessary means to complete the project successfully.

I also try to arouse the students' curiosity by proposing a subject that is more playful than usual.

Following these objectives, I propose a self-working magic card trick. The aim of the course/project is to synthetize this card trick with an open source digital electronics simulator.

Consequently, the students have to look for, find and understand a 'new' theory of digital electronics and new software: Logisim.

Finally, I try to change the traditional way of teaching: a downward method (the professor teaches the student) usually used in France, become an ascending method here (it is the student who will have to explain to the professor).

The ascending method has the merit of showing whether the problem posed is well understood. As Boileau wrote (Boileau, 1740) "An idea well conceived presents itself clearly, and the words to express it come readily"1

\section{Materials and Methods: Using the Self-Working Card Trick Based On the Cyclic Number $142857(1 / 7=0.142857)$}

I summarize in this part the magic trick and the main results we have found in the previous part. I think it is essential to remember you the project and how you can teach with a down-top pedagogy.

\subsection{The Self-Working Card Trick Effect}

"Prepare a deck of playing cards by removing the nine spades and nine hearts with digit values. Place them on the bottom of the deck so that their order from the bottom up is $1,4,2,8,5,7$, with the remaining three cards following in any order. Place away the 6 selected hearts as a prediction [...] A random multiplier from 1 through 6 is now obtained by rolling a die. Better still, hand someone an imaginary die and ask him to roll this invisible die and tell you what he "sees" on top. Multiply 142,857 by the digit he names. Take and then cut the prediction deck (in order to determine where to cut, multiply 7 by the selected digit to get the last digit of the product)." (Gardner, 1992).

\subsection{The Mathematical Secret}

"The number 142,857, which students of recreational number theory are likely to recognize at once, is one of the most remarkable of integers. Apart from the trivial case of 1 , it is the smallest "cyclic number." A cyclic number is an integer of $n$ digits with an unusual property: when multiplied by any number from 1 through n, the product contains the same $n$ digits of the original number in the same cyclic order. Think of 142,857 as being joined end to end in a circular chain. The circle can be broken at six points and the chain can be opened to form six digit numbers, the six cyclic permutations of the original digits:
$1 \times 142857=142857$
$2 \times 142857=285714$
$3 \times 142857=428571$
$4 \times 142857=571428$
$5 \times 142857=714285$
$6 \times 142857=857142$

1 "ce qui se conçoit bien s'énonce clairement, et les mots pour le dire arrivent aisément". 
The cyclic nature of the six products has long intrigued magicians, and many clever mathematical prediction tricks are based on it (Regnault, 1943) (Costa, 1953) (Schott, 2016). How to teach the sequential part of digital electronics basis with project pedagogy? Thanks to a self-working card trick named "cyclic number"! IESJR, 2(9). Simon (1964). Here is one:" (Gardner, 1992).

To find this kind of number with the same properties, take the prime number, divide it by 1 and if the digit number of the perodic decimal part is equal to the number minus one, then you have found a new cyclic number! Here $1 / 7=0, \mathbf{1 4 2 8 5 7} 142587 \ldots$

\subsection{Global Study and How to Cut In Pedagogical Sequences with Ascending Pedagogy}

The first sequence! Firstly I perform the magic trick and then I propose the students to realize it ... using digital electronics (with seven segments displays) ... but the students have to figure it out! Secondly, and it's the aim of this first sequence, consists in breaking up this large problem into smaller bits. It should achieve the anticipated outcome: to find smaller unity problems (but students have to think, they had "alone" find the solutions) and potentially in the opposite order shown in the "normal" top-down pedagogical method:

- Using a cycle (142857) with different connections to achieve the project;

- Several representations of a single number (coming from a card or the dice) are needed, hence the introduction of binary code and balanced code;

- How to give rhythm to the cycle? We introduce here flip flops as part of using up-counter and downcounter;

- I personally don't want to use any of these counters but a very special cycle so I have to synthetize control circuits. So I have to introduce combinatory logic;

- In order to create these control circuits, we have to make functions, hence the introduction of the SYSTEM's truth tables and the use of Karnaugh maps;

- But then we need to know some elementary gates to synthetize with gates the results of the Karnaugh maps approach.

- At the end, I have to display the numbers in order to perform the magic show. That's why, we have to study the seven segment displays with the associated encoders and decoders! But we have 13 displays and we have to select one of them thus the MUX and DEMUX circuits must be used.

So, all of the needed notions are found and the next step can be reached. Each week, the students have to find the theoretical notions for each single problem then find a solution and finally implement it. The teacher work consists "only" to be sure that the theoretical found notions by the students are right and that the students have well understood them! If necessary, the teacher can (must) expose and explain the notions with the found informations; that's very important that the teacher use ONLY the found notions... the figure shows the complete digital circuit for the 142857 magic trick.

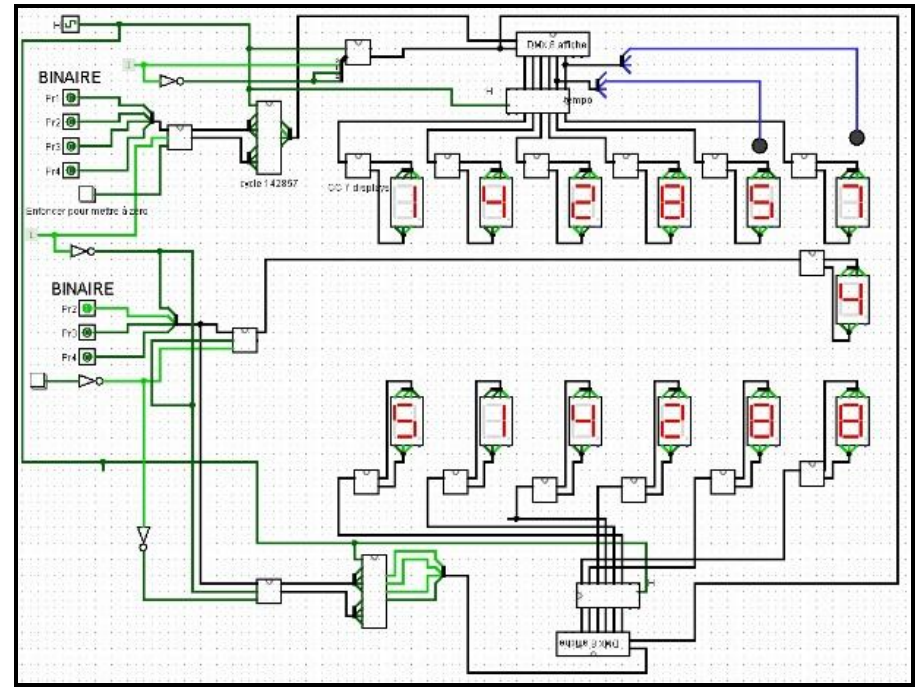

Figure-1. The complete 142857 digital electronics circuit.

\subsection{Project Representation by States}

The representation of the project is shown on this figure

Figure-: 


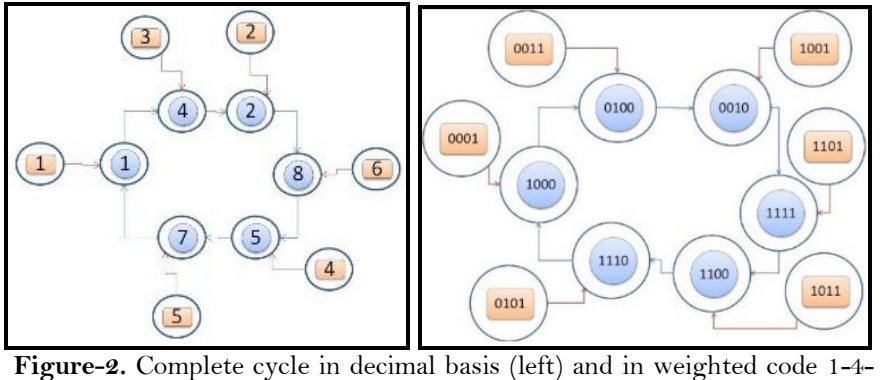

2-1 of the cyclic used number 142857 .

\subsection{Conclusion of the Study}

We have to synthetize 4 macros circuits:

- a transcoder binary -> weighted code;

- a "transcoder" weighted code $->$ the display;

- a multiplexer to select a 7 segment displays;

- a synchronous or asynchronous counter with many command circuits before the each flip-flop gate of the counter.

Only the last macro circuit has not yet been explained!

\section{Results}

In order to synthetize all kind of counter, first of all, you have to define the counter's structure: synchronous or asynchronous counters, flip-flop or D-latch gates, aso. The find the transition table is necessary to synthetize the command circuits of each sequential gates. At the end, testing the final circuit is strength recommended!

\subsection{The Number of the Flip-Flop Gates}

We actually count how many states we have. Here we have 12 states and the aim is to find n so $2^{\mathrm{n}-1}<12$ $\leq 2^{n}$. Thus $n=4$ and it's the number of flip-flop gates that we needed for a synchronous or asynchronous counter.

\subsection{The Used Structure of This Counter}

To move from one element of the cycle to the next one, the inputs of the sequential gates must be changed. In fact a NEW logic states (1 or 0 ) of the flipflops outputs can be reach on each rising edge (or falling edge) of the global clock. The circuits which calculate the new inputs of the sequential gates depending to the past element of the cycle are named: control circuit (CCJiKi). The goal is to synthesize these circuits. It is the classic case of a combinatorial system synthesis, as seen before BUT the inputs are the binary word which describes one state of the cycle and the outputs are the inputs of the sequential gates. The global structure of the circuit is shown on figure.

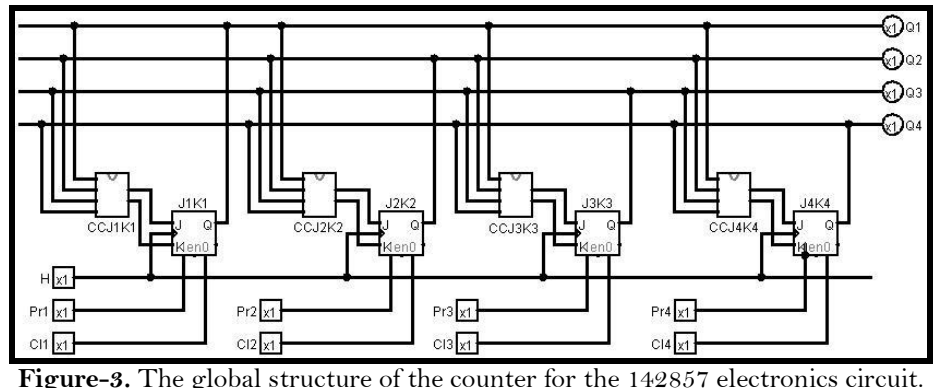

The Main Important To Synthesize a “Counter”: The Transition Table

In order to synthesize the CCJiKi you have to know the transition table. It is construct by using the laws of the JK flip-flop written as follows:

- If the output is equal to $\mathrm{O}$ and after the clockrise the output must be equal to $\mathrm{O}$, then $\mathrm{J}=\mathrm{O}$ and $\mathrm{K}=\mathrm{x}$;

- If the output is equal to 1 and after the clockrise the output must be equal to 0 , then $\mathrm{J}=\mathrm{x}$ and $\mathrm{K}=1$;

- If the output is equal to $\mathrm{O}$ and after the clockrise the output must be equal to 1 , then $\mathrm{J}=1$ and $\mathrm{K}=\mathrm{x}$;

- If the output is equal to 1 and after the clock rise the output must be equal to 1 , then $\mathrm{J}=\mathrm{x}$ and $\mathrm{K}=\mathrm{O}$. 
I develop an Excel sheet in order to automatically find the system transition table. The only thing you have to do is to complete the binary words before and the binary word after the clock rise. The result for the 142857 application is shown on figure

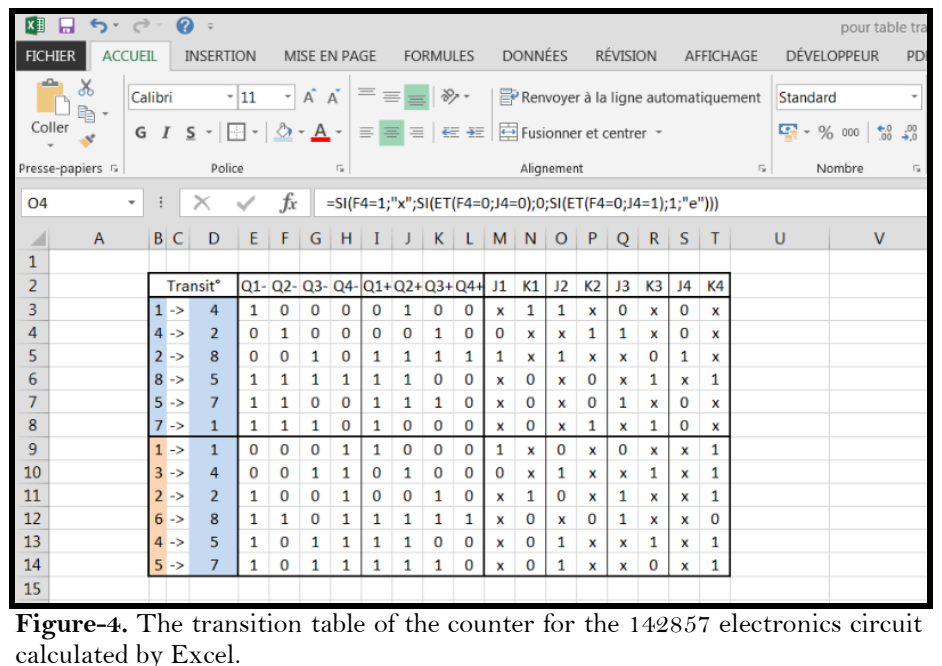

Thanks to Karnaugh's table, the CCJiKi can be easily synthetized. Two differents set of circuits can be found:

- the first set is obtained when the transition of unused binary words is not defined (i.e. the $\mathrm{Ji}=\mathrm{Ki}=\mathrm{x}$ );

- the second set is obtained when the transition of unused binary goes to the beginning of the cycle (i.e. the "1" state).

\section{Discussion}

It's easily understandable to teach Math or Optics Illusion with magic. In this paper, I have proposed to teach sequential part of the digital electronic using Magic and the experience shows that it works quiet good!

I think that it seems easy to tech as explained but if you have never used the down-top pedagogy, you have:

- $\quad$ to give a reading packet which the students must fill up. At the end, they will have a whole project report!

- $\quad$ to write on your preparation paper all of the needed notions you have to introduce.

- to have a notebook in which you put down all the interactions you have with each (group of) student(s).

In order to begin a such pedagogy, you can introduce a part of you course with this pedagogy the first year; the second year, you can teach the half program and the so on.

\section{Acknowledgments}

This paper could not have been written without the encouragements of Bernard SCHOTT, my father, the invaluable help of Anne SERRIE, my mother, Claire LEROUX, the head of ARNUM.

\section{References}

Boileau, N. (1740). Scholasticism. Paris: Souchay.

Costa, A. (1953). The cyclic number 142857. Scripta Mathematica, 19, 181-184.

Gardner, M. (1992). Mathematical circus (pp. 111-114). Washington, DC: The Mathematical Association of America.

Regnault, D. (1943). Les calculateurs prodiges (pp. 336-343). Paris: Payot.

Schott, P. (2016). How to teach the sequential part of digital electronics basis with project pedagogy? Thanks to a selfworking card trick named cyclic number. IESJR, 2(9).

Simon, W. (1964). A slate prediction trick based on 142,857 (pp. 31-35): Mathematical Magic, Scribner's. 